\title{
Nonsingular Terminal Sliding Mode Control of Uncertain Second-Order Nonlinear Systems
}

\author{
Minh-Duc Tran' and Hee-Jun Kang ${ }^{2}$ \\ ${ }^{1}$ University of Ulsan, Ulsan 680-749, Republic of Korea \\ ${ }^{2}$ School of Electrical Engineering, University of Ulsan, Ulsan 680-749, Republic of Korea \\ Correspondence should be addressed to Hee-Jun Kang; hjkang@ulsan.ac.kr
}

Received 27 April 2015; Revised 10 July 2015; Accepted 13 July 2015

Academic Editor: Rongwei Guo

Copyright (c) 2015 M.-D. Tran and H.-J. Kang. This is an open access article distributed under the Creative Commons Attribution License, which permits unrestricted use, distribution, and reproduction in any medium, provided the original work is properly cited.

\begin{abstract}
This paper presents a high-performance nonsingular terminal sliding mode control method for uncertain second-order nonlinear systems. First, a nonsingular terminal sliding mode surface is introduced to eliminate the singularity problem that exists in conventional terminal sliding mode control. By using this method, the system not only can guarantee that the tracking errors reach the reference value in a finite time with high-precision tracking performance but also can overcome the complex-value and the restrictions of the exponent (the exponent should be fractional number with an odd numerator and an odd denominator) in traditional terminal sliding mode. Then, in order to eliminate the chattering phenomenon, a super-twisting higher-order nonsingular terminal sliding mode control method is proposed. The stability of the closed-loop system is established using the Lyapunov theory. Finally, simulation results are presented to illustrate the effectiveness of the proposed method.
\end{abstract}

\section{Introduction}

As the development of control schemes has progressed, a variety of control systems have been developed for robotic manipulators, including proportional-integral-derivative (PID) control [1], adaptive control [2], computed torque control [3,4], fuzzy control [5], and neural network control [6]. Sliding mode control (SMC) is an efficient control method that has been widely applied to control for both linear and nonlinear systems. In order to design sliding mode control systems, establishment of suitable sliding surfaces to ensure the desired dynamics is considered first, and then a sliding mode controller is designed to drive the system states to the sliding surface. The main characteristic of SMC is to use discontinuous control effort to keep the system states on the sliding surfaces, whereby SMC has strong robustness with respect to system uncertainties and external disturbances, fast response, and good transient performance. However, the conventional SMC method cannot guarantee the invariance properties during the reaching phase and even against disturbances can degrade the performance of system [7-9]. Moreover, this method adopts a linear sliding surface, which can only provide asymptotic stability of the system in the sliding phase.

Terminal sliding mode control (TSMC) methods, which use nonlinear sliding surfaces instead of a linear surface, were first introduced by Venkataraman and Gulati [10] and further developed by Man et al. [11, 12] and Wu et al. [13]. Compared with linear SMC, TSMC schemes not only ensure that the system states arrive at the equilibrium point in a finite time but also offer some attractive properties, such as their fast response and higher precision. However, the traditional TSMC methods may have slower convergence performance when the system states are not near the equilibrium point, and they also suffer from the singularity problem and have restrictions on the range of the power function. In order to avoid these drawbacks, some new TSMC methods have been proposed [14-16]. Yu and Zhihong [14] have developed fast terminal sliding mode (FTSM), which can improve the convergence speed when the system states are far from the equilibrium point. This method, however, still has the singularity problem. To overcome this, Feng et al. [16] introduced nonsingular terminal sliding mode (NTSM) control. 
However, this surface has a limitation on the power function; that is, $p$ and $q$ must be positive odd integers.

Discontinuous terminal sliding mode control (TSMC) has been widely applied to nonlinear systems. Nevertheless, the main drawback of discontinuous TSMC is the chattering phenomenon, which comes from high frequency switching of the control signal. It shows undesirable oscillation on the system, leads to low control accuracy, causes high wear of the moving mechanical parts, and may damage the actuators. To deal with this problem, the most common methods replace the sign function in the switching control with a saturating approximation [17] or boundary layer technique [18]. The boundary layer method was proposed to eliminate the chattering by defining a boundary layer around the sliding surface and then approximate the discontinuous control by continuous function within this boundary layer. As a result, the chattering elimination is achieved; however, there is a trade-off between chattering elimination and tracking performance; a thicker boundary layer can eliminate the chattering phenomenon but the tracking error will be increased. Recently, intelligent control schemes (neural network and fuzzy logic) have been applied to attenuate the chattering phenomenon [19-21]. However, some controller designs based on intelligence techniques were quite complicated and fell into difficulties in stability analysis. Therefore, in this study, high-order sliding mode (HOSM) techniques have been studied and applied. The main characteristic of HOSM is that they are working with the discontinuous control in the higher-order time derivative [22-27], so the chattering can be reduced because the control signal is continuous. Furthermore, HOSM can bring better accuracy than conventional SMC while the robustness of the control system is similar to SMC. It has been presented in [23-25] for the control of rigid robot manipulators.

In this paper, the above-mentioned problems are addressed based on a proposed NTSM surface for second-order nonlinear systems. A control law is designed to drive the system states to reach the sliding surface and converge to zero in a finite time. It does not suffer from the singularity problem or the restriction on the power function. Furthermore, a super-twisting second-order sliding mode is also used to reduce the chattering of the controller. The global finite time stability of the closed-loop system is proven. The convergence times of the reaching phase and sliding phase are also given. The simulation results are presented to illustrate the effectiveness of the proposed method on the two-link robot manipulator.

The remainder of this paper is arranged as follows. Preliminaries and problem formulation are given in Section 2. In Section 3, the structure of super-twisting nonsingular terminal sliding mode controller is presented and a stability analysis is performed. In Section 4, simulation results for a two-link robot manipulator are provided to demonstrate the performance of the proposed controller. Finally, some concluding remarks are presented in Section 5.

\section{Preliminaries and Problem Formulation}

Consider the following nonlinear second-order mechanical systems:

$$
\begin{aligned}
& \dot{x}_{1}=x_{2}, \\
& \dot{x}_{2}=f(x, t)+d(x, t)+b(x, t) u(t),
\end{aligned}
$$

where $x=\left[x_{1}, x_{2}\right]^{T}$ denotes the system state vector, $f(x, t)$ and $b(x, t)$ are smooth nonlinear functions of $x, u(t)$ is the control input, and $d(x, t)$ presents the uncertainties and disturbances.

Assumption 1. The matrices $b(x, t)$ are invertible $\forall x$.

Assumption 2. The uncertain term is bounded by

$$
|d(x, t)| \leq \bar{D}
$$

where $\bar{D}$ is a known positive constant.

Assumption 3. The desired state vector $x_{d}(t) \in R$ is a twice continuously differentiable function in terms of $t$.

The control objective of this paper is to design a controller for system (1) to ensure that the error between the real state vector $x$ and the desired state vector $x_{d}(t)$ converges to zero in finite time.

\section{Main Results}

In this section, the design of super-twisting nonsingular terminal sliding mode controller is presented. First, a new nonsingular terminal sliding mode surface is proposed to eliminate the singularity problem. Then, the conventional SMC and super-twisting nonsingular terminal sliding mode controller are designed to ensure that the tracking error converges to zero in a finite amount time.

3.1. New Form of NTSM Surface. We define the tracking error as $\varepsilon(t)=x_{1}(t)-x_{1 d}(t)$. Thus, a new NTSM surface is proposed as follows:

$$
s=\dot{\varepsilon}+\beta_{1} \varepsilon+\beta_{2} e^{-\lambda t}\left(\varepsilon^{T} \varepsilon\right)^{-\alpha} \varepsilon,
$$

where $s=\left[s_{1}, s_{2}, \ldots, s_{n}\right]^{T}, \varepsilon=\left[\varepsilon_{1}, \varepsilon_{2}, \ldots, \varepsilon_{n}\right]^{T}, \dot{\varepsilon}=\left[\dot{\varepsilon}_{1}\right.$, $\left.\dot{\varepsilon}_{2}, \ldots, \dot{\varepsilon}_{n}\right]^{T}, \beta_{1}=\operatorname{diag}\left(\beta_{11}, \beta_{12}, \ldots, \beta_{1 n}\right), \beta_{2}=\operatorname{diag}\left(\beta_{21}\right.$, $\beta_{22}, \ldots, \beta_{2 n}$ ) with $\beta_{1 i}, \beta_{2 i}>0$ for every $i=1,2, \ldots, n, 0<$ $\alpha<1$, and $\lambda>0$. is true:

When the system operates in sliding mode, the following

$$
\begin{aligned}
& s=\dot{\varepsilon}+\beta_{1} \varepsilon+\beta_{2} e^{-\lambda t}\left(\varepsilon^{T} \varepsilon\right)^{-\alpha} \varepsilon=0 \\
& \dot{\varepsilon}=-\beta_{1} \varepsilon-\beta_{2} e^{-\lambda t}\left(\varepsilon^{T} \varepsilon\right)^{-\alpha} \varepsilon .
\end{aligned}
$$

Theorem 4. Considering the sliding mode dynamic equation (5), the system is finite time stable at the equilibrium point $\varepsilon=$ 0 , and the tracking error $\varepsilon$ will converge to zero in finite time if $2 \alpha \lambda_{\min }\left(\beta_{1}\right)-\lambda>0$. 
The finite convergence time is

$$
T_{s} \leq \frac{\ln \left(1+\left(e^{2 \alpha \lambda_{\min }\left(\beta_{1}\right) t} \cdot V^{\alpha}(0)\right) / a_{2}\right)}{2 \alpha \lambda_{\min }\left(\beta_{1}\right)-\lambda},
$$

where $a_{2}$ is expressed by (15).

Proof. Consider the Lyapunov function:

$$
V=\frac{1}{2} \varepsilon^{T} \varepsilon
$$

Taking the derivative of $V$ in (7) and substituting (5) into it yield

$$
\begin{aligned}
\dot{V} & =\varepsilon^{T} \dot{\varepsilon}=\varepsilon^{T}\left[-\beta_{1} \varepsilon-\beta_{2} e^{-\lambda t}\left(\varepsilon^{T} \varepsilon\right)^{-\alpha} \varepsilon\right], \\
\dot{V} & =-\varepsilon^{T} \beta_{1} \varepsilon-\beta_{2} e^{-\lambda t}\left(\varepsilon^{T} \varepsilon\right)^{-\alpha} \varepsilon^{T} \varepsilon \\
& \leq-\lambda_{\min }\left(\beta_{1}\right) \varepsilon^{T} \varepsilon-\lambda_{\min }\left(\beta_{2}\right) e^{-\lambda t}\left(\varepsilon^{T} \varepsilon\right)^{1-\alpha} \\
& \leq-2 \lambda_{\min }\left(\beta_{1}\right) V-2^{1-\alpha} \lambda_{\min }\left(\beta_{2}\right) e^{-\lambda t} V^{1-\alpha} \leq 0 .
\end{aligned}
$$

Therefore, according to the Lyapunov stability, it is obvious that the origin is at globally stable equilibrium. Next, we will show that the system states converge to zero in finite time.

Multiplying both sides of (9) by $\alpha V^{\alpha-1}$, we have

$$
\begin{aligned}
& \alpha V^{\alpha-1} \frac{d V}{d t} \leq-2 \alpha \lambda_{\min }\left(\beta_{1}\right) V^{\alpha}-2^{1-\alpha} \alpha \lambda_{\min }\left(\beta_{2}\right) e^{-\lambda t}, \\
& \frac{d V^{\alpha}}{d t}+2 \alpha \lambda_{\min }\left(\beta_{1}\right) V^{\alpha} \leq-2^{1-\alpha} \alpha \lambda_{\min }\left(\beta_{2}\right) e^{-\lambda t} .
\end{aligned}
$$

Multiplying both sides of (10) by $e^{2 \alpha \lambda_{\min }\left(\beta_{1}\right) t}$ yields

$$
\begin{aligned}
& e^{2 \alpha \lambda_{\min }\left(\beta_{1}\right) t}\left(\frac{d V^{\alpha}}{d t}+2 \alpha \lambda_{\min }\left(\beta_{1}\right) V^{\alpha}\right) \\
& \leq-2^{1-\alpha} \alpha \lambda_{\min }\left(\beta_{2}\right) e^{\left[2 \alpha \lambda_{\min }\left(\beta_{1}\right)-\lambda\right] t}, \\
& \frac{d\left(e^{2 \alpha \lambda_{\min }\left(\beta_{1}\right) t} \cdot V^{\alpha}\right)}{d t} \leq-2^{1-\alpha} \alpha \lambda_{\text {min }}\left(\beta_{2}\right) e^{\left[2 \alpha \lambda_{\min }\left(\beta_{1}\right)-\lambda\right] t} .
\end{aligned}
$$

Taking the integral on both sides of (12) from 0 to $T_{s}$ and knowing $V\left(T_{s}\right)=0$ yield

$$
\begin{aligned}
-e^{2 \alpha \lambda_{\min }\left(\beta_{1}\right) t} \cdot V^{\alpha}(0) & \leq-a_{2}\left[e^{\left[2 \alpha \lambda_{\min }\left(\beta_{1}\right)-\lambda\right] T_{s}}-1\right], \\
e^{\left[2 \alpha \lambda_{\min }\left(\beta_{1}\right)-\lambda\right] T_{s}} & \leq 1+\frac{e^{2 \alpha \lambda_{\min }\left(\beta_{1}\right) t} \cdot V^{\alpha}(0)}{a_{2}},
\end{aligned}
$$

where

$$
a_{2}=\frac{2^{1-\alpha} \alpha \lambda_{\min }\left(\beta_{2}\right)}{2 \alpha \lambda_{\min }\left(\beta_{1}\right)-\lambda}>0 .
$$

Taking the natural logarithm of both sides of (14) yields

$$
\begin{aligned}
& {\left[2 \alpha \lambda_{\min }\left(\beta_{1}\right)-\lambda\right] T_{s} \leq \ln \left(1+\frac{e^{2 \alpha \lambda_{\min }\left(\beta_{1}\right) t} \cdot V^{\alpha}(0)}{a_{2}}\right) .} \\
& T_{s} \leq \frac{\ln \left(1+\left(e^{2 \alpha \lambda_{\min }\left(\beta_{1}\right) t} \cdot V^{\alpha}(0)\right) / a_{2}\right)}{2 \alpha \lambda_{\min }\left(\beta_{1}\right)-\lambda}
\end{aligned}
$$

This completes the proof.
Remark 5. The expression in (3) is different from the previously reported TSM and fast TSM in [14], which are expressed, respectively, as

$$
\begin{aligned}
& s=\dot{x}+\beta x^{q / p}, \\
& s=\dot{x}+\alpha x+\beta x^{q / p},
\end{aligned}
$$

where $\alpha$ and $\beta$ are positive constants and $p$ and $q$ are positive odd integers that satisfy the following condition: $1<p / q<2$. We can easily see that, for $x<0$, the fractional power $q / p$ may lead to the term $x^{q / p} \notin R$, which means $\dot{x} \notin R$. In addition, the TSM control signals in [14] contain $x_{1}^{q / p-1} x_{2}$, which may cause a singularity to occur if $x_{2} \neq 0$ when $x_{1}=0$.

To solve the complex-value problem in (17), Yu et al. [28] proposed the TSM surface as

$$
\begin{aligned}
& s=\dot{x}+\beta|x|^{\gamma} \operatorname{sign}(x), \\
& s=\dot{x}+\alpha x+\beta|x|^{\gamma} \operatorname{sign}(x) .
\end{aligned}
$$

The sliding surface in (18) could solve the complex-value number, but the control input can suffer from the singularity problem if $x_{2} \neq 0$ when $x_{1}=0$.

Recently, a nonsingular terminal sliding surface was proposed to overcome the singularity problem [16]:

$$
s=x+\frac{1}{\beta} \dot{x}^{p / q} \text {. }
$$

However, this surface still has the limitation for the exponent of the power function; that is, $p$ and $q$ should be positive odd integers. Thus, our proposed TSM surface does not contain any of the mentioned singularities, and the exponent can be any real number in the interval $0<\alpha<1$.

Remark 6. Comparing with linear sliding mode, NTSM has higher convergence rate when the system state is far away from the equilibrium point, while NTSM has lower convergence speed when the system state is close to the equilibrium point $[29,30]$.

It is obvious that the term $e^{-\lambda t}$ in the proposed surface will go backward to zero after a certain time. Thus, the nonsingular terminal sliding mode surface will become linear sliding mode after a period of time. By choosing a suitable $\lambda$, the proposed surface will have the advantage of both NTMS and linear sliding surface.

3.2. NTSM Control (NTSMC) Design. One suitable sliding manifold is established. The next step is to design the control to drive the nonlinear system (1) to the expected sliding surface (3) in a finite amount time. The proposed control method is summarized as follows.

Theorem 7. For the system (1), if the control signal is designed as (20) and the gain $\eta$ of the controller is larger than the upper 
bounds of the uncertainties, the tracking error $\varepsilon(t)$ will converge to zero in finite time:

$$
\begin{aligned}
u(t)= & -b(x, t)^{-1} \\
& \cdot\left[f(x, t)-\ddot{x}_{d}+\beta_{1} \dot{\varepsilon}+\beta_{2} A+\eta \operatorname{sign}(s)\right],
\end{aligned}
$$

where $\eta=\operatorname{diag}\left(\eta_{1}, \eta_{2}, \ldots, \eta_{n}\right), \eta_{i}>0$. Therefore,

$$
\begin{aligned}
A & =\left[(-\lambda) e^{-\lambda t}\left(\varepsilon^{T} \varepsilon\right)^{-\alpha} \varepsilon\right. \\
& \left.+e^{-\lambda t}(-2 \alpha) x\left(\varepsilon^{T} \varepsilon\right)^{-\alpha-1}\left(\varepsilon^{T} \dot{\varepsilon}\right) \varepsilon+e^{-\lambda t}\left(\varepsilon^{T} \varepsilon\right)^{-\alpha} \dot{\varepsilon}\right] .
\end{aligned}
$$

Proof. Consider the following Lyapunov candidate function:

$$
V=\frac{1}{2} s^{T} s
$$

The time derivative of the sliding surface (3) with respect to time can be expressed as

$$
\begin{aligned}
\dot{\mathcal{s}}= & \ddot{\varepsilon}+\beta_{1} \dot{\varepsilon}+\beta_{2} A \\
= & f(x, t)+d(x, t)+b(x, t) u(t)-\ddot{x}_{d}(t)+\beta_{1} \dot{\varepsilon} \\
& +\beta_{2} A .
\end{aligned}
$$

Differentiating $V$ with respect to time and substituting (20) and (23) into it yield

$$
\dot{V}=s^{T}(-\eta \operatorname{sign}(s)+d(t)) \leq-(\eta-\bar{D})|s| \leq 0 .
$$

Therefore, the condition for Lyapunov stability is satisfied; in the following, we will show that the error converges to zero in finite time.

From (24), we have

$$
\begin{aligned}
& \dot{V} \leq-\sqrt{2}(\eta-\bar{D}) V^{1 / 2} \\
& d t \leq-\frac{d V}{\sqrt{2}(\eta-\bar{D}) V^{1 / 2}}=-\frac{\sqrt{2} d V^{1 / 2}}{(\eta-\bar{D})} .
\end{aligned}
$$
have

Taking the integral of both sides of (25) from $T_{r}$ to $T_{s}$, we

$$
T_{s}-T_{r} \leq-\int_{V\left(T_{r}\right)}^{V\left(T_{s}\right)} \frac{\sqrt{2} d V^{1 / 2}}{(\eta-\bar{D})}=\frac{\sqrt{2}}{(\eta-\bar{D})} V^{1 / 2}\left(T_{r}\right)
$$

Note that $V\left(T_{s}\right)=0$; therefore, the TSM will reach zero in the finite time:

$$
T_{s} \leq \frac{\sqrt{2}}{(\eta-\bar{D})} V^{1 / 2}\left(T_{r}\right)+T_{r}
$$

This completes the proof.

Remark 8. In order to eliminate the chattering, a saturation function sat or $s /(\|s\|+\varepsilon)$ ( $\varepsilon$ is a small positive constant) can be used to replace the sign function.
3.3. Super-Twisting NTSM Control (ST-NTSMC) Design. The main drawback of the conventional sliding mode is the chattering phenomenon which is caused by discontinuous control action when the system state operates near the sliding surface. Even though the chattering reduction can be achieved by using Remark 8 , there is a trade-off between chattering elimination and tracking performance; increasing the thickness of the boundary layer can eliminate the chattering phenomenon but will increase the tracking error. Therefore, in this subsection, super-twisting control is applied to attenuate chattering and to increase the tracking performance.

The ST-NSTSMC is designed as

$$
u=u_{\mathrm{eq}}+u_{\mathrm{STW}}
$$

where

$$
u_{\mathrm{eq}}=-b(x, t)^{-1}\left[f(x, t)-\ddot{x}_{d}+\beta_{1} \dot{\varepsilon}+\beta_{2} A\right] .
$$

Based on [27], the super-twisting controller is designed as

$$
\begin{aligned}
u_{\mathrm{STW}} & =-b(x, t)^{-1}\left(k_{1}|s|^{1 / 2} \operatorname{sign}(s)+z\right), \\
\dot{z} & =-k_{2} \operatorname{sign}(s) .
\end{aligned}
$$

The differentiation of the sliding surface is now obtained as

$$
\begin{aligned}
\dot{s}= & f(x, t)+d(x, t)+b(x, t)\left(u_{\mathrm{eq}}+u_{\mathrm{SMW}}\right)-\ddot{x}_{d}(t) \\
& +\beta_{1} \dot{\varepsilon}+\beta_{2} A .
\end{aligned}
$$

Substituting (29) and (30) into (31) yields

$$
\begin{aligned}
& \dot{s}=-k_{1}|s|^{1 / 2} \operatorname{sign}(s)+z+d(x, t), \\
& \dot{z}=-k_{2} \operatorname{sign}(s) .
\end{aligned}
$$

The stability and convergence of the closed-loop system in (32) are given in Theorem 9.

Theorem 9. Suppose that Assumption 1 is guaranteed and the uncertain terms are bounded by

$$
\begin{aligned}
& d(x, t) \leq \delta|s|^{1 / 2}, \\
& \delta=\operatorname{diag}\left(\delta_{1}, \delta_{2}, \ldots, \delta_{n}\right), \quad \delta_{i}>0 .
\end{aligned}
$$

For system (1), with the terminal sliding mode surface chosen as in (3) and the proposed control signal designed as in (28), if the sliding gains of $u_{\text {STW }}$ given in (30) satisfy condition (34), then the sliding surface $s$ will converge to zero in a finite time:

$$
\begin{aligned}
& k_{1}>2 \delta, \\
& k_{2}>k_{1} \frac{5 k_{1}+4 \delta}{2\left(k_{1}-2 \delta\right)} \delta .
\end{aligned}
$$

Proof. Now, referring to Moreno's work [27], let us consider the Lyapunov candidate function:

$$
V=\xi^{T} P \xi
$$


where

$$
\begin{aligned}
& \xi=\left[|s|^{1 / 2} \operatorname{sign}(s), z\right]^{T}, \\
& P=\frac{1}{2}\left[\begin{array}{cc}
k_{1}{ }^{2}+4 k_{2} & -k_{1} \\
-k_{1} & 2
\end{array}\right] .
\end{aligned}
$$

As we know, $V$ is positive definite and radially unbounded:

$$
\lambda_{\min }(P)\|\zeta\|^{2} \leq V \leq \lambda_{\max }(P)\|\zeta\|^{2},
$$

where $\|\zeta\|^{2}=|s|+z^{2}$. The time derivative of $V$ becomes

$$
\dot{V}=-\frac{1}{|s|^{1 / 2}}\left(\xi^{T} Q_{1} \xi-d(x, t) Q_{2}{ }^{T} \xi\right),
$$

where

$$
\begin{aligned}
Q_{1} & =\frac{k_{1}}{2}\left[\begin{array}{cc}
k_{1}^{2}+2 k_{2} & -k_{1} \\
-k_{1} & 1
\end{array}\right], \\
Q_{2}{ }^{T} & =\left[\frac{k_{1}^{2}}{2}+2 k_{2}-\frac{k_{1}}{2}\right] .
\end{aligned}
$$

Using condition (33), it can be shown that

$$
\dot{V} \leq-\frac{1}{|s|^{1 / 2}} \xi^{T} Q \xi \leq-\frac{1}{|s|^{1 / 2}} \lambda_{\min }(Q)\|\xi\|^{2},
$$

where

$$
Q=\frac{k_{1}}{2}\left[\begin{array}{cc}
k_{1}^{2}+2 k_{2}-\left(\frac{4 k_{2}}{k_{1}}+k_{1}\right) \delta & -\left(k_{1}+2 \delta\right) \\
-\left(k_{1}+2 \delta\right) & 1
\end{array}\right] .
$$

In the case in which the condition in (34) is satisfied, $Q>$ 0 , so $\dot{V}$ is negative definite.

We can use (37) and the fact that

$$
\begin{gathered}
|s|^{1 / 2} \leq\|\zeta\| \leq \frac{V^{1 / 2}}{\lambda_{\min }{ }^{1 / 2}(P)} \\
\|\zeta\| \geq \frac{V^{1 / 2}}{\lambda_{\max }^{1 / 2}(P)} .
\end{gathered}
$$

Then, substituting (42) into (40) yields

$$
\dot{V} \leq-\kappa V^{1 / 2}
$$

where

$$
\kappa=\frac{\lambda_{\min }^{1 / 2}(P) \lambda_{\min }(Q)}{\lambda_{\max }(P)} .
$$

Since the solution of the differential equation

$$
\begin{aligned}
\dot{v} & \leq-\kappa v^{1 / 2}, \\
v(0) & =v_{0}>0
\end{aligned}
$$

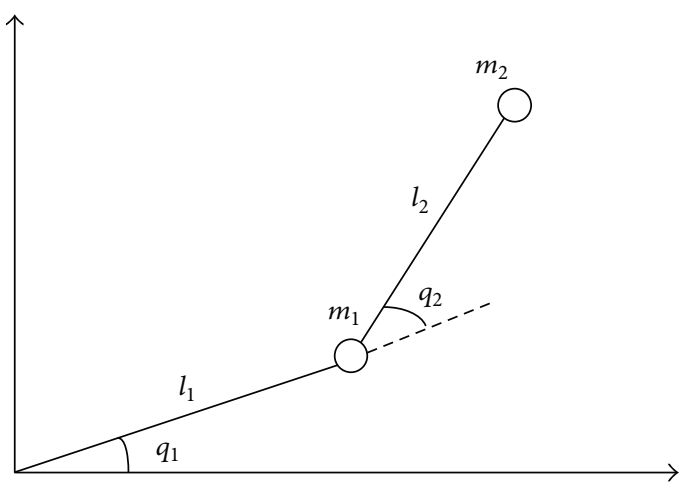

FIgURE 1: Configuration of the two-link robotic system [3].

is given as

$$
v(t)=\left(v_{0}^{1 / 2}-\frac{\kappa}{2} t\right)^{2},
$$

here, $v(t)$ converges to zero in a finite time and reaches zero after $T=2 V^{1 / 2}\left(x_{0}\right) / \kappa$. It follows from the comparison principle [18] that $V(t) \leq v(t)$ when $V\left(x_{0}\right) \leq v_{0}$. From (46), we can determine that $V(t)$ and therefore $s$ converge to zero in a finite time and reach that value at most after $T=2 V^{1 / 2}\left(x_{0}\right) / \kappa$.

\section{Simulation Results}

In this section, to verify the validity and effectiveness of the proposed method, the two-link planar robot manipulator shown in Figure 1 is considered.

The dynamic equation of the two-link robot is described as follows [3]:

$$
M(q) \ddot{q}+C(q, \dot{q})+G(q)=\tau(t)+\tau_{d}+F(\dot{q}),
$$

where

$$
\begin{aligned}
& M(q) \\
& =\left[\begin{array}{cc}
l_{2}^{2} m_{2}+2 l_{1} l_{2} m_{2} c_{2}+l_{1}^{2}\left(m_{1}+m_{2}\right) & l_{2}^{2} m_{2}+l_{1} l_{2} m_{2} c_{2} \\
l_{2}^{2} m_{2}+l_{1} l_{2} m_{2} c_{2} & l_{2}^{2} m_{2}
\end{array}\right], \\
& C(q, \dot{q})=\left[\begin{array}{c}
-m_{2} l_{1} l_{2} s_{2} \dot{q}_{2}^{2}-2 m_{2} l_{1} l_{2} s_{2} \dot{q}_{1} \dot{q}_{2} \\
m_{2} l_{1} l_{2} s_{2} \dot{q}_{1}^{2}
\end{array}\right], \\
& G(q)=\left[\begin{array}{c}
m_{2} l_{2} g c_{12}+\left(m_{1}+m_{2}\right) l_{1} g c_{1} \\
m_{2} l_{2} g c_{12}
\end{array}\right],
\end{aligned}
$$

and $q=\left(q_{1}, q_{2}\right)^{T}$ is the joint variable vector, $M(q)$ is the inertial matrix, $C(q, \dot{q})$ represents the centripetal and Coriolis torque matrix, $G(q)$ represents the gravity torque vector, $\tau_{d}$ is the vector of the bounded external disturbance, $F(\dot{q})$ is the friction, and $\tau$ is the control torque. $m_{1}$ and $m_{2}$ are the link masses, $l_{1}$ and $l_{2}$ are the link lengths, gravity $g=9.81\left(\mathrm{~m} / \mathrm{s}^{2}\right)$, and the symbols $s_{1}, s_{2}, s_{12}$ and $c_{1}, c_{2}, c_{12}$ are, respectively, defined as $s_{1}=\sin \left(q_{1}\right), s_{2}=\sin \left(q_{2}\right), s_{12}=\sin \left(q_{12}\right), c_{1}=$ $\cos \left(q_{1}\right), c_{2}=\cos \left(q_{2}\right)$, and $c_{12}=\cos \left(q_{12}\right)$. 


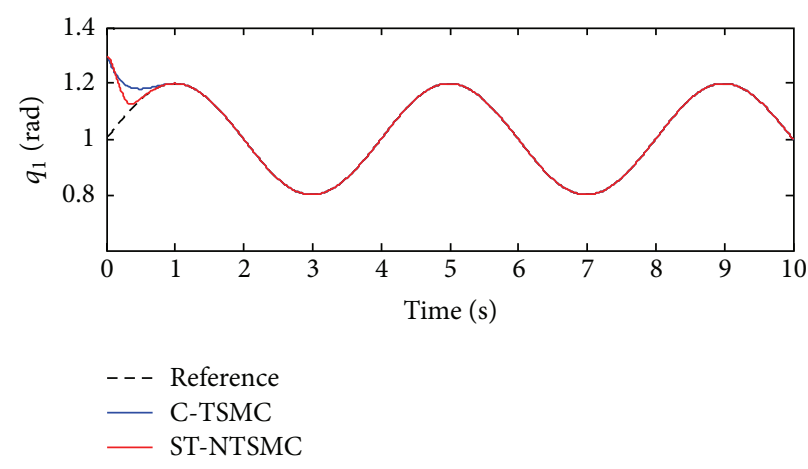

(a)

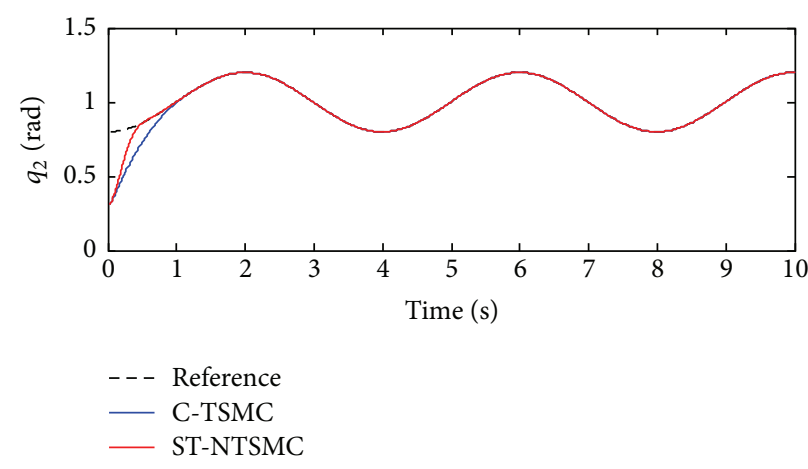

(b)

FIGURE 2: Tracking performance of two-link robot manipulator, (a) at joint 1, (b) at joint 2.

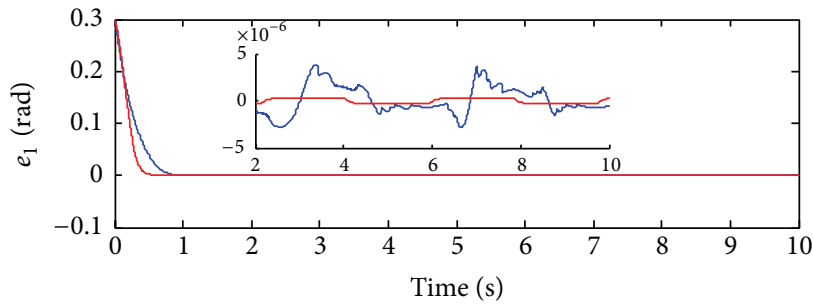

C-TSMC

- ST-NTSMC

(a)

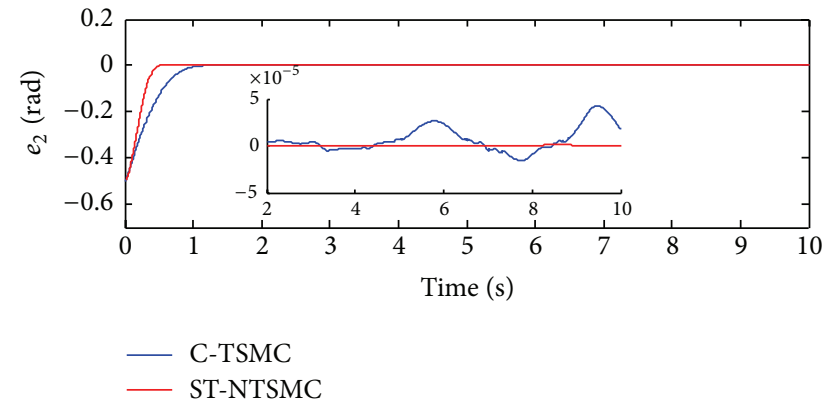

(b)

FIgURE 3: Tracking errors of two-link robot manipulator, (a) at joint 1, (b) at joint 2.

The friction and external disturbance are chosen as

$$
\begin{aligned}
F(\dot{q}) & =\left[\begin{array}{c}
\dot{q}_{1}+2 \sin \left(q_{1}\right) \\
0.5 \sin \left(q_{2}\right)
\end{array}\right], \\
\tau_{d} & =\left[\begin{array}{c}
0.2 \sin (t) \\
0.2 \cos (2 t)
\end{array}\right] .
\end{aligned}
$$

The parameter values employed to simulate the robot are given as $m_{1}=m_{2}=1(\mathrm{~m})$ and $l_{1}=l_{2}=1(\mathrm{~kg})$, and the design reference signals are given by

$$
\begin{aligned}
& q_{1 d}=1+0.2 \sin (0.5 \pi t), \\
& q_{2 d}=1-0.2 \cos (0.5 \pi t) .
\end{aligned}
$$

The initial states of the system are chosen as

$$
\begin{aligned}
& q_{1}(0)=1.3, \\
& q_{2}(0)=0.3, \\
& \dot{q}_{1}(0)=0, \\
& \dot{q}_{2}(0)=0 .
\end{aligned}
$$

To this end, Matlab/Simulink is used to perform all of the simulations, and with the sampling time set to $10^{-4} \mathrm{~s}$, the simulation compares the proposed ST-NTSMC control
TABLE 1: Control parameters.

\begin{tabular}{ll}
\hline Control schemes & Parameters \\
\hline C-TSMC [28] & $\beta=10 I_{2}, \eta_{1}=25 I_{2}, \eta_{2}=45 I_{2}, \gamma=1.5, \varphi=0.3$. \\
ST-NTSMC & $\begin{array}{l}\beta_{1}=12 I_{2}, \beta_{2}=10 I_{2}, k_{1}=9, k_{2}=5, \lambda=3, \\
\\
\alpha=0.3 .\end{array}$ \\
\hline
\end{tabular}

scheme with the previously proposed control method in [28]. Yu et al. [28] suggested the continuous terminal sliding mode control (C-TSMC), which was designed for a two-link robot manipulator as follows:

$$
\begin{aligned}
\tau= & C_{0}(q, \dot{q})+G_{0}(q)+M_{0}(q) \ddot{q}_{d} \\
& -M_{0} \beta^{-1} \gamma^{-1} \operatorname{sig}(\dot{e})^{(2-\gamma)}-M_{0}\left(\eta_{1} s+\eta_{2} \operatorname{sig}(s)^{\varphi}\right),
\end{aligned}
$$

where $s=e+\beta \operatorname{sig}(\dot{e})^{\gamma}, \operatorname{sig}(x)^{\gamma}=\left[\left|x_{1}\right|^{\gamma} \operatorname{sign}\left(x_{1}\right)\right.$, $\left.\left|x_{2}\right|^{\gamma} \operatorname{sign}\left(x_{2}\right), \ldots,\left|x_{n}\right|^{\gamma} \operatorname{sign}\left(x_{n}\right)\right], \beta=\operatorname{diag}\left(\beta_{1}, \beta_{2}, \ldots, \beta_{n}\right)$, $\eta_{1}=\operatorname{diag}\left(\eta_{11}, \eta_{12}, \ldots, \eta_{1 n}\right), \eta_{2}=\operatorname{diag}\left(\eta_{21}, \eta_{22}, \ldots, \eta_{2 n}\right), \beta_{i}$, $\eta_{1 i}, \eta_{2 i}>0,0<\varphi<1$, and $1<\gamma<2$.

The control parameters are selected as shown in Table 1.

The simulation results are shown in Figures 2-5. In Figure 2, the tracking results of the robot manipulator using the two control laws above are compared. It shows that the state trajectories can reach the design reference signals in the presence of model parameter uncertainties and external 


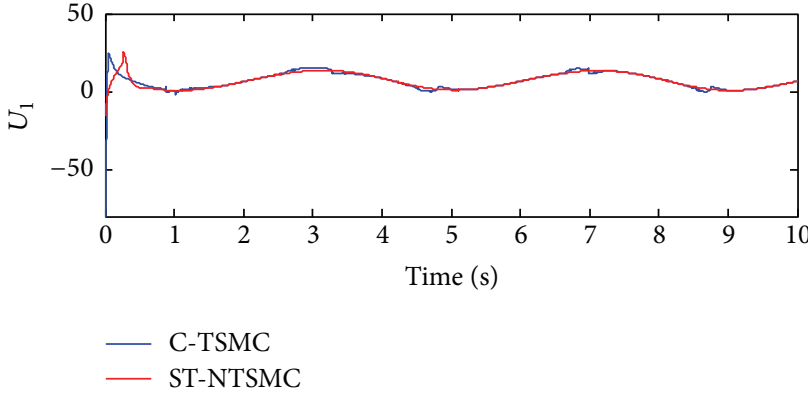

(a)

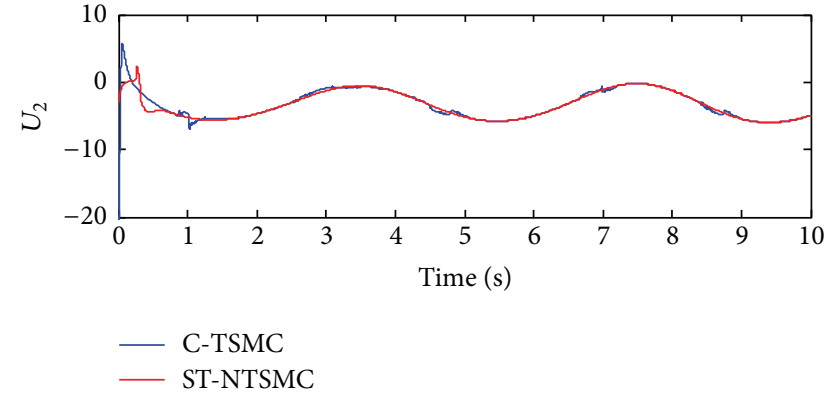

(b)

FIGURE 4: Control inputs, (a) at joint 1, (b) at joint 2.

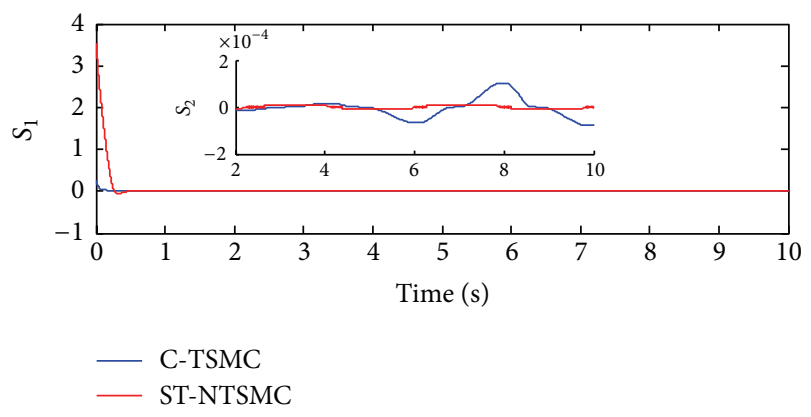

(a)

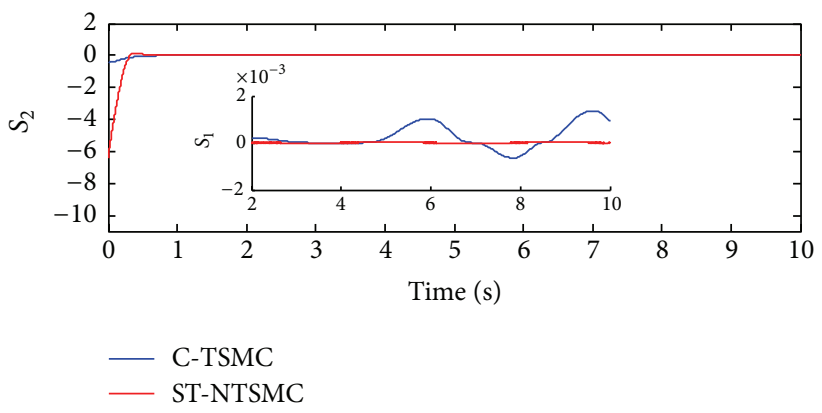

(b)

FIGURE 5: Time responses of the terminal sliding mode surface, (a) at joint 1 , (b) at joint 2.

disturbances. The tracking errors via two controllers are compared in Figure 3. One can easily see that the ST-NTSMC produces tracking performance with faster convergence and higher precision. Figure 4 shows the time histories of the applied control inputs and shows that the proposed STNTSMC method achieves superior control input performance with smaller control efforts, higher precision tracking, and smoother than the C-TSMC method. The time responses of the sliding manifolds are shown in Figure 5. Clearly, the sliding surface of the proposed method was also much smaller than C-TSMC.

\section{Conclusions}

In this paper, we presented the ST-NTSMC method for second-order nonlinear systems. This method has been successfully applied in a two-link robot manipulator. The designed nonsingular terminal sliding surface not only avoids the singularity problem, but also can overcome the complexvalue and the restriction on the exponent of a power function in conventional TSMC. The performance of the proposed method was evaluated in comparison with recently proposed approaches [28]. The simulation results show that the proposed method achieves highly precise tracking, fast and finite time convergence, and robustness against parameter uncertainties and external disturbances. Furthermore, STNTSMC is used to smooth the discontinuous control term in order to attenuate the chattering phenomenon.

\section{Conflict of Interests}

The authors declare that there is no conflict of interests regarding the publication of this paper.

\section{Acknowledgment}

This paper is a result of a study on the "Leaders IndustryUniversity Cooperation" Project, supported by the Ministry of Education (MOE).

\section{References}

[1] S. Arimoto and F. Miyazaki, "Stability and robustness of PID feedback control for robot manipulators of sensory capability," in Robotic Research, M. Brady and R. P. Paul, Eds., MIT Press, Cambridge, Mass, USA, 1984.

[2] J.-J. E. Slotine and W. Li, "On the adaptive control of robot manipulators," International Journal of Robotics Research, vol. 6, no. 3, pp. 49-59, 1987.

[3] J. J. Craig, Introduction to Robotics, Addion-Wesley, Reading, Mass, USA, 1989.

[4] J. J. Spong and M. Vidyasagar, Robot Dynamics and Control, Wiley, New York, NY, USA, 1989.

[5] J. H. Lilly, Fuzzy Control and Identification, Wiley, 2010.

[6] L. Jinkun, Radial Basis Function Neural Network Control for Mechanical Systems, Tsinghua University Press, Beijing, China, 2013. 
[7] V. I. Utkin, "Variable structure systems with sliding modes," IEEE Transactions on Automatic Control, vol. 22, no. 2, pp. 212222, 1977.

[8] J. Y. Hung, W. Gao, and J. C. Hung, "Variable structure control: a survey," IEEE Transactions on Industrial Electronics, vol. 40, no. 1, pp. 2-22, 1993.

[9] A. Šabanovic, "Variable structure systems with sliding modes in motion control-a survey," IEEE Transactions on Industrial Informatics, vol. 7, no. 2, pp. 212-223, 2011.

[10] S. T. Venkataraman and S. Gulati, "Control of nonlinear systems using terminal sliding modes," Transactions of the ASMEJournal of Dynamic Systems, Measurement and Control, vol. 115, no. 3, pp. 554-560, 1993.

[11] Z. Man, A. P. Paplinski, and H. R. Wu, "A robust MIMO terminal sliding mode control scheme for rigid robotic manipulators," IEEE Transactions on Automatic Control, vol. 39, no. 12, pp. 2464-2469, 1994.

[12] Z. Man and X. Yu, "Terminal sliding mode control of MIMO linear systems," IEEE Transactions on Circuits and Systems. I. Fundamental Theory and Applications, vol. 44, no. 11, pp. 10651070, 1997.

[13] Y. Wu, X. Yu, and Z. Man, "Terminal sliding mode control design for uncertain dynamic systems," Systems \& Control Letters, vol. 34, no. 5, pp. 281-287, 1998.

[14] X. Yu and M. Zhihong, "Fast terminal sliding-mode control design for nonlinear dynamical systems," IEEE Transactions on Circuits and Systems. I. Fundamental Theory and Applications, vol. 49, no. 2, pp. 261-264, 2002.

[15] L. Yang and J. Yang, "Nonsingular fast terminal sliding-mode control for nonlinear dynamical systems," International Journal of Robust and Nonlinear Control, vol. 21, no. 16, pp. 1865-1879, 2011.

[16] Y. Feng, X. Yu, and Z. Man, "Non-singular terminal sliding mode control of rigid manipulators," Automatica, vol. 38, no. 12, pp. 2159-2167, 2002.

[17] T. H. S. Li and Y.-C. Huang, "MIMO adaptive fuzzy terminal sliding-mode controller for robotic manipulators," Information Sciences, vol. 180, no. 23, pp. 4641-4660, 2010.

[18] J. J. E. Slotine and W. Li, Applied Nonlinear Control, PrenticeHall, Englewood Cliffs, NJ, USA, 1991.

[19] Y. Jiang, Q. Wang, and C. Dong, "A reaching law neural network terminal sliding mode guidance law design," in Proceedings of the IEEE Region 10 Conference (TENCON '13), Xi'an, China, October 2013.

[20] B. Yoo and W. Ham, "Adaptive fuzzy sliding mode control of nonlinear system," IEEE Transactions on Fuzzy Systems, vol. 6, no. 2, pp. 315-321, 1998.

[21] M. Roopaei, M. Zolghadri Jahromi, and S. Jafari, "Adaptive gain fuzzy sliding mode control for the synchronization of nonlinear chaotic gyros," Chaos, vol. 19, no. 1, Article ID 013125, 2009.

[22] J. Rivera, C. Mora, J. J. Raygoza, and S. Ortega, "Suppertwisting sliding mode in motion control systems," in Sliding Mode Control, A. Bartoszewicz, Ed., pp. 978-953, InTech, 2011.

[23] D. Hernandez, W. Yu, and M. A. Moreno-Amendariz, "Neural PD control with second-order sliding mode compensation for robot manipulators," in Proceedings of the International Joint Conference on Neural Networks (IJCNN '11), pp. 2392-2402, San Jose, Calif, USA, August 2011.

[24] L. M. Capisani, A. Ferrara, and L. Magnani, "Second order sliding mode motion control of rigid robot manipulators," in Proceedings of the 46th IEEE Conference on Decision and Control (CDC '07), pp. 3691-3696, December 2007.
[25] M. Van, H.-J. Kang, and Y.-S. Suh, "Second order sliding mode-based output feedback tracking control for uncertain robot manipulators," International Journal of Advanced Robotic Systems, vol. 10, article 16, 2013.

[26] J. Davila, L. Fridman, and A. Levant, "Second-order slidingmode observer for mechanical systems," IEEE Transactions on Automatic Control, vol. 50, no. 11, pp. 1785-1789, 2005.

[27] J. A. Moreno and M. Osorio, "A Lyapunov approach to secondorder sliding mode controllers and observers," in Proceedings of the 47th IEEE Conference on Decision and Control, pp. 28562861, December 2008.

[28] S. Yu, X. Yu, B. Shirinzadeh, and Z. Man, "Continuous finitetime control for robotic manipulators with terminal sliding mode," Automatica, vol. 41, no. 11, pp. 1957-1964, 2005.

[29] X. Zhao, Y. X. Jiang, Y. J. Wu, and Y. Q. Zhou, "Fast nonsingular terminal sliding mode control based on multi-slide-mode," Journal of Beijing University of Aeronautics and Astronautics, vol. 37, no. 1, pp. 110-113, 2011.

[30] H. J. Shi, L. F. Qian, Y. D. Xu, and L. M. Chen, "Fuzzy moving fast terminal sliding mode control for robotic manipulators," in Proceedings of the IEEE International Conference on Robotics and Biomimetics (ROBIO '12), pp. 1943-1949, IEEE, Guangzhou, China, December 2012. 


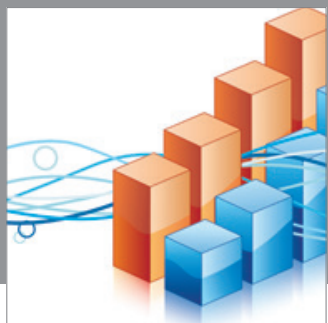

Advances in

Operations Research

mansans

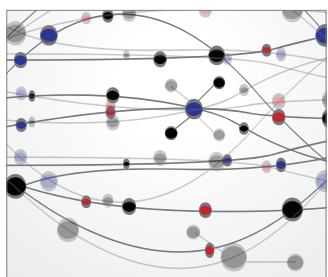

The Scientific World Journal
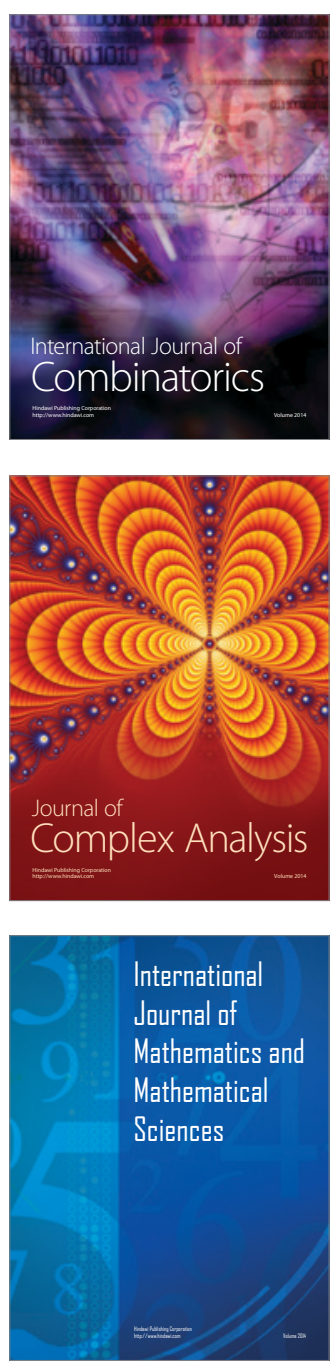
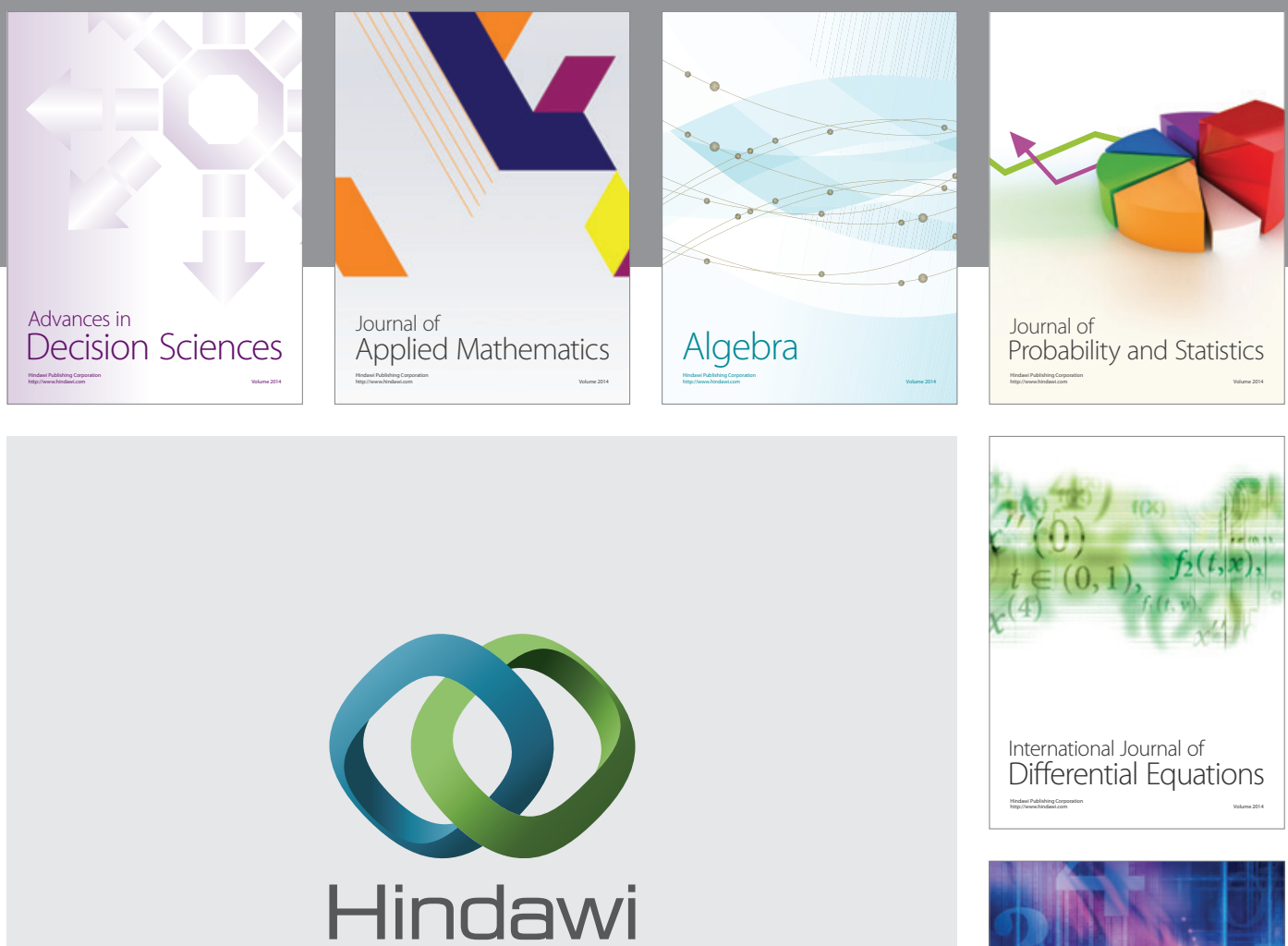

Submit your manuscripts at http://www.hindawi.com
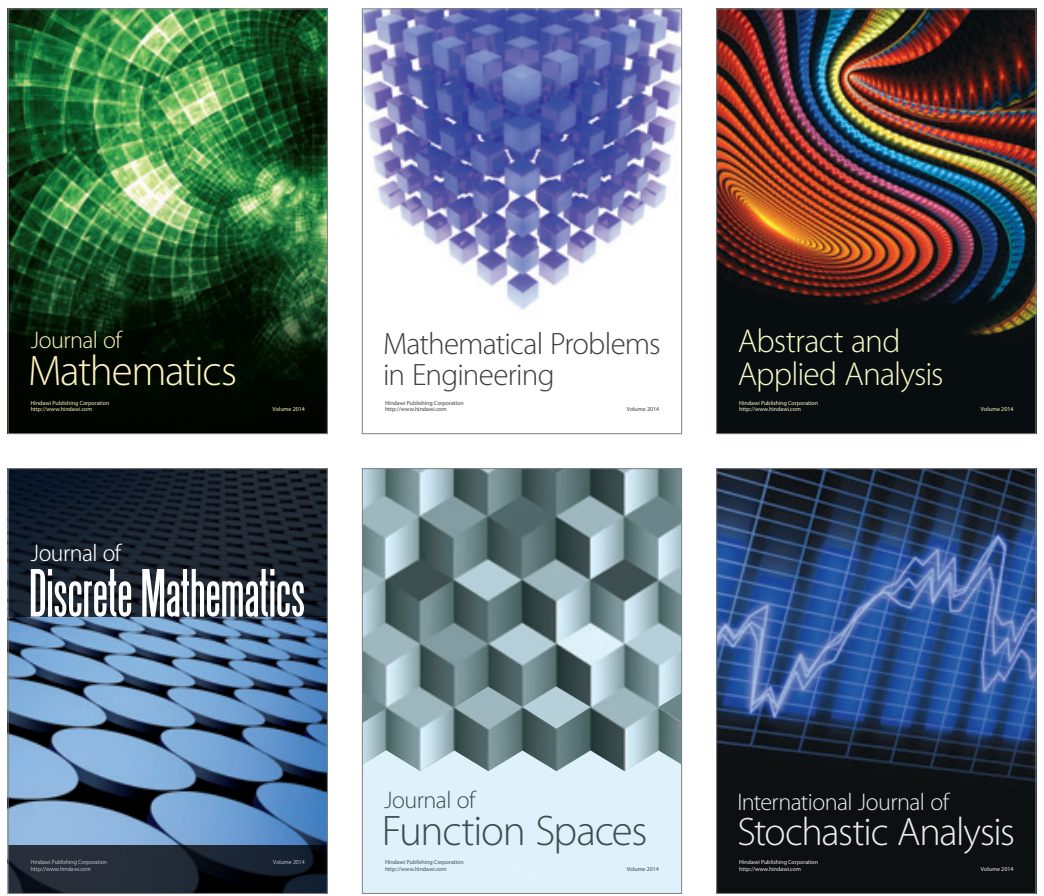

Journal of

Function Spaces

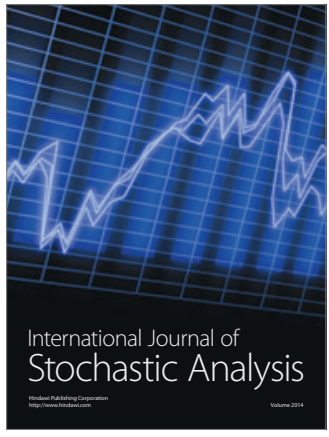

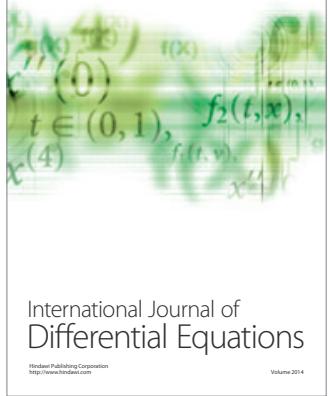
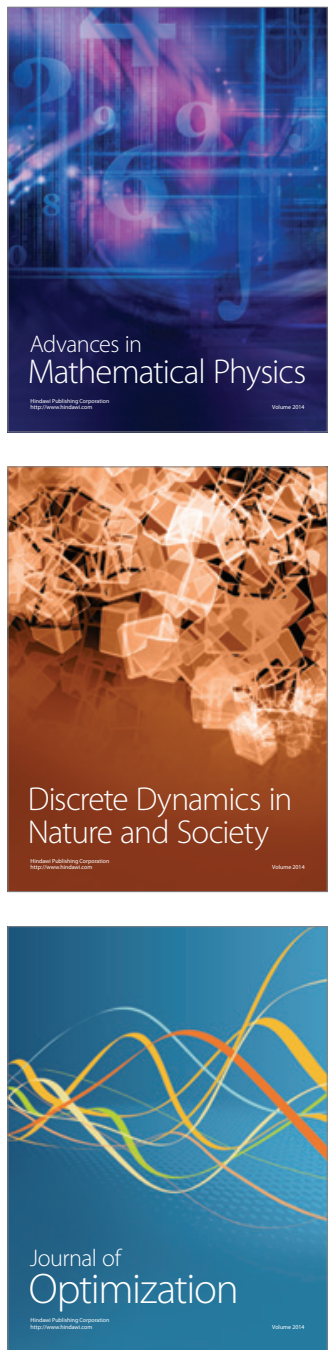